\title{
Cancer-associated immunodeficiency and dendritic cell abnormalities mediated by the prostaglandin EP2 receptor
}

\author{
Li Yang, ${ }^{1,2,3}$ Noboru Yamagata, ,2,3 Rajwardhan Yadav, ${ }^{4}$ Suzanne Brandon,, 5 \\ Regina L. Courtney, ${ }^{1,5}$ Jason D. Morrow, ${ }^{1,5}$ Yu Shyr, ${ }^{6}$ Mark Boothby, ${ }^{4}$ Sebastian Joyce, ${ }^{4}$ \\ David P. Carbone, ${ }^{1,2,3}$ and Richard M. Breyer ${ }^{1,3,5}$ \\ ${ }^{1}$ Department of Medicine, \\ ${ }^{2}$ Department of Cancer Biology, \\ ${ }^{3}$ The Vanderbilt-Ingram Cancer Center, \\ ${ }^{4}$ Department of Microbiology and Immunology, \\ ${ }^{5}$ Department of Pharmacology, and \\ ${ }^{6}$ Department of Preventative Medicine, Vanderbilt University School of Medicine, Nashville, Tennessee, USA
}

Prostaglandin $\mathrm{E}_{2}\left(\mathrm{PGE}_{2}\right)$, a major COX metabolite, plays important roles in several facets of tumor biology. We characterized the contribution of the $\mathrm{PGE}_{2} \mathrm{EP} 2$ receptor to cancer-associated immune deficiency using $E P 2^{-/-}$mice. $E P 2^{-/-}$mice exhibited significantly attenuated tumor growth and longer survival times when challenged with MC26 or Lewis lung carcinoma cell lines as compared with their wild-type littermates. While no differences in $\mathrm{T}$ cell function were observed, $\mathrm{PGE}_{2}$ suppressed differentiation of DCs from wild-type bone marrow progenitors, whereas EP2-null cells were refractory to this effect. Stimulation of cells in mixed lymphocyte reactions by wild-type DCs was suppressed by treatment with $\mathrm{PGE}_{2}$, while $E P 2^{-/-}$-derived DCs were resistant to this effect. In vivo, DCs, $\mathrm{CD}^{+}$, and $\mathrm{CD8}^{+} \mathrm{T}$ cells were significantly more abundant in draining lymph nodes of tumor-bearing $\mathrm{EP}^{-/-}$mice than in tumor-bearing wild-type mice, and a significant antitumor cytotoxic $\mathrm{T}$ lymphocyte response could be observed only in the EP2-/- animals. Our data demonstrate an important role for the EP2 receptor in $\mathrm{PGE}_{2}$-induced inhibition of DC differentiation and function and the diminished antitumor cellular immune responses in vivo.

J. Clin. Invest. 111:727-735 (2003). doi:10.1172/JCI200316492.

\section{Introduction}

Tumor-induced immune suppression is a fundamental problem in cancer biology and immunotherapy. COX metabolites act as tumor promoters when overproduced (1-3), and recent studies have demonstrated that the COX metabolite prostaglandin $\mathrm{E}_{2}\left(\mathrm{PGE}_{2}\right)$ exhibits potent immunosuppressive effects, orchestrating an imbalance between type 1 and type 2 cytokines (4-6). $\mathrm{PGE}_{2}$ mediates its effects, in part, through $\mathrm{G}$ proteincoupled PGE receptors, designated EP1, EP2, EP3, and EP4. Differential expression of these EP receptors mediate the diverse, and often antagonistic, effects of $\mathrm{PGE}_{2}$ and its analogues on a variety of cell types (7-9). The EP2 receptor regulates the activation and differentiation of mouse B lymphocytes (10), modulates $\mathrm{T}$ cell

Received for publication July 24, 2002, and accepted in revised form January 7, 2003.

Address correspondence to: Richard M. Breyer, Vanderbilt University, Division of Nephrology, 116121 st Avenue South Nashville, Tennessee 37232-2372, USA. Phone: (615) 343-0257; Fax: (615) 343-4704; E-mail: rich.breyer@vanderbilt.edu.

David P. Carbone and Richard M. Breyer contributed equally to this work.

Conflict of interest: The authors have declared that no conflict of interest exists.

Nonstandard abbreviations used: prostaglandin $\mathrm{E}_{2}\left(\mathrm{PGE}_{2}\right) ; \mathrm{T}$ cell receptor (TCR); phycoerythrin (PE); mixed lymphocyte reaction (MLR); cytotoxic T lymphocyte (CTL). development (11-12), and regulates macrophage cytokine release (13) and postsurgery immune responses (14). The EP2 receptor also plays a key role in the differentiation of macrophage-like osteoclast cells as well as the functional response of osteoclasts to $\mathrm{PGE}_{2}$ (15). Importantly, $\mathrm{PGE}_{2}$ has been shown to be a key modulator of DC function, altering cytokine production as well as the I-Ad class II cell surface marker (16-17). Despite data describing the production of $\mathrm{PGE}_{2}$ by tumors and the expression of the $\mathrm{EP} 2$ receptor in specific immune populations, the role of the EP2 receptor in modulation of the host immune response to tumors remains uncharacterized.

Previous studies show that COX-2 and $\mathrm{PGE}_{2}$ can play important roles in tumor angiogenesis $(3,18$, 19). Recent publications show that in $A p c^{\Delta 716}$ mice, a mouse model for human familial adenomatous polyposis, homozygous deletion of the gene encoding EP2 decreases the number and size of intestinal polyps through inhibition of tumor angiogenesis (19, 20). In this study we determined the role of the EP2 receptor in host-tumor interactions. Unlike the results observed in the $A p c^{\Delta 716}$ model, we observed no effect of the disruption of the EP2 receptor on tumor angiogenesis. We then examined the role of the EP2 receptor in $\mathrm{T}$ cell function, as well as DC differentiation, and functional responses to tumor challenge. Our data demonstrate an important role for the EP2 
receptor in $\mathrm{PGE}_{2}$-induced inhibition of DC differentiation and function and as a mediator of cancerassociated immunodeficiency.

\section{Methods}

Mice. EP2 $2^{-/-}$mice were generated as described (21) and backcrossed for ten generations onto both the $\mathrm{C} 57 \mathrm{BL} / 6$ and the BALB/c background strains. Eightto ten-week-old female mice used in the study were housed in pathogen-free units at Vanderbilt University School of Medicine, in compliance with Institutional Animal Care and Use Committee regulations. EP2-null $\mathrm{BALB} / \mathrm{c}$ mice were crossed with $\mathrm{BALB} / \mathrm{c}$ with a transgenic $T$ cell receptor (TCR) specific for the ovalbumin peptide OVA 323-339 (22).

Reagents. Ab-producing hybridomas were obtained from the American Type Culture Collection (Manassas, Virginia, USA) and used as culture supernatants: anti-CD4 (L3T4, TIB-207), anti-CD8 (Lyt-2.2, TIB-210), and anti-MHC class II (TIB-120). Mouse GM-CSF was obtained from R\&D Systems Inc. (Minneapolis, Minnesota, USA). Anti-CD3, anti-CD28, and anti-CD69 were obtained from PharMingen (San Diego, California, USA). FITC-conjugated antiCD11c was purchased from PharMingen. Isotypematched FITC-conjugated IgG was used as a control of nonspecific binding. $\mathrm{PGE}_{2}$ is from Cayman Chemical (Ann Arbor, Michigan, USA). FITC-conjugated or phycoerythrin-conjugated (PE-conjugated) Ab's were purchased from PharMingen.

Cell culture. The MC26 colorectal cancer cell line, Lewis lung carcinoma cell line, 3LL, and R8, an Abelson leukemia virus-transformed cell line, as well as RMA-S cells, were maintained according to standard cell-culture techniques.

RT-PCR analysis of EP2 receptor expression in bone marrow progenitors. Total RNA was extracted from bone marrow progenitors of $\mathrm{BALB} / \mathrm{c}-E P 2^{-/-}$and wild-type mice $\left(E P 2^{+/+}\right)$with an RNeasy Mini Kit (QIAGEN Inc., Valencia, California, USA). Primers specific for the EP2 receptor (sense: 5'-CCGGGGTTCTGGGGAATC-3'; antisense: $5^{\prime}$-AACCTCAGGACCGGTGGC-3') were used in RT-PCR analysis.

Bone marrow-derived DC culture and FACs analysis. Bone marrow was obtained from the femurs and tibias of BALB/c-EP2 $2^{--}$and wild-type mice. The cells were then enriched for hematopoietic cells by depletion of lineage-specific cells with a mixture of Ab's (TIB-207, TIB-210, and TIB-120) and complement (Cedarlane Laboratories Ltd., Hornby, Ontario, Canada) and subsequent gradient-density centrifugation on Lympholyte M (Cedarlane Laboratories Ltd.) to remove dead cells. Progenitors were cultured in GM-CSF-containing medium to generate DCs. $\mathrm{PGE}_{2}$ or vehicle was added to the DC cell cultures every $24 \mathrm{~h}$. DCs were harvested and washed in PBS and labeled with anti-CD11c and other Ab's for 30 minutes at $4{ }^{\circ} \mathrm{C}$. To investigate the number of DCs and $\mathrm{CD}^{+}$and $\mathrm{CD}^{+} \mathrm{T}$ cells in draining lymph nodes of tumor-bearing mice, single-cell suspensions of inguinal lymph nodes from tumor-bearing $\mathrm{EP}^{-{ }^{--}}$ and wild-type mice were prepared 21 days after MC26 inoculation, the time at which we observed the maximum tumor growth difference between $E P 2^{-/-}$and wild-type control mice. Cells were labeled with PEconjugated anti-H2IA ${ }^{d}$, anti-CD11c, anti-CD4, or anti-CD8 Ab's, washed, and analyzed on a FACScan flow cytometer (Becton Dickinson Immunocytometry Systems, Mountain View, California, USA).

Measurements of $T$ cell cytokine production. CD4 T cells from lymph nodes of EP2 wild-type or EP2-null animals were enriched by complement-mediated lysis using anti-CD8 anti-MHC class II and then stimulated to differentiate to Th1 or Th2 cells as described (23). Briefly, cells were incubated with immobilized anti-CD3, anti-CD28 Ab's, and recombinant mouse IL-2, in the presence of neutralizing polyclonal $\mathrm{Ab}$ against mouse IL-4 (for Th1) or IL-12 (for Th2), as appropriate. Washed cells (day 6) were restimulated with immobilized anti-CD3 plus anti-CD28 for $24 \mathrm{~h}$ before collection of culture supernatants for determination of cytokine production. Cytokine (IL-4 and IFN- $\gamma$ ) levels from culture supernatants were determined by standard ELISA according to the manufacturer's instructions (PharMingen).

Measurements of $P G E_{2}$ concentration. Draining lymph nodes, tumor tissues, and serum of tumor-bearing mice were collected, and their purification, derivation, and analysis of $\mathrm{PGE}_{2}$ by gas chromatography/mass spectrometry were performed as described (24-25).

$T$ cell proliferation. For alloreactive mixed lymphocyte reaction (MLR), single-cell suspensions were prepared from inguinal, axillary, and brachial lymph nodes of wild-type C57BL/6 mice. Red cells were removed by hypotonic shock. T cells were enriched using hybridoma supernatant (TIB 120, anti-class II; American Type Culture Collection) and the complement depletion method followed by enrichment by Lympholyte M gradient-density centrifugation for $20 \mathrm{~min}$ at $600 \mathrm{~g}$. T cells were collected from the interface, washed, and used in the MLR assay. Day 9 DCs from BALB/c-EP2-/- and wild-type mice, treated with or without $\mathrm{PGE}_{2}$, were irradiated with $20 \mathrm{~Gy}$, plated in triplicate, and incubated with allogeneic $\mathrm{T}$ cells for $72 \mathrm{~h}$. Eighteen hours before harvesting, all cultures were pulsed with $\left[{ }^{3} \mathrm{H}\right]$ thymidine (Amersham Life Sciences Inc., Arlington Heights, Illinois, USA). Incorporation of $\left[{ }^{3} \mathrm{H}\right]$ thymidine was counted using a liquid scintillation counter and expressed as counts per minute.

For peptide-induced proliferation, day 9 bone marrow-derived DCs $\left(10^{5}\right)$ from wild-type BALB/c mice were irradiated and pulsed with varying concentrations of OVA 323-339 peptide for $2 \mathrm{~h}$. T cells $\left(4 \times 10^{5}\right)$, prepared as described above from the lymph nodes and spleen of DO11.10/EP2 $2^{-/}$, DO11.10/EP2 ${ }^{+/}$, or DO.11-10/EP2 ${ }^{+/+}$ transgenic mice (BALB/c background), were then cocultured for $72 \mathrm{~h}$. Eighteen hours before harvesting, all cultures were pulsed with $\left[{ }^{3} \mathrm{H}\right]$ thymidine (Amersham Life 
Sciences Inc.). Incorporation of $\left[{ }^{3} \mathrm{H}\right]$ thymidine was counted using a liquid scintillation counter and expressed as counts per minute.

${ }^{51}$ Cr-release cytotoxic T lymphocyte assay. BALB $/ \mathrm{c}-E P 2^{-/-}$ and wild-type mice were immunized two times by intraperitoneal injection with $2 \times 10^{7}$ irradiated MC26 cells $\left(\mathrm{H} 2 \mathrm{~K}^{\mathrm{d}}\right)$. Spleen cells from MC26-immunized mice were restimulated in vitro with irradiated MC26 cells for 4 days in a mixed leukocyte-tumor cell culture. Responder cells were harvested and used at different ratios in a cytotoxic T lymphocyte (CTL) assay using ${ }^{51} \mathrm{Cr}$-labeled MC26 as target cells. After $6 \mathrm{~h}$, supernatants were harvested, and the released radioactivity was measured. $\mathrm{R} 8$, an $\mathrm{H} 2^{\mathrm{b} / \mathrm{d}}$ cell line, was used as a specificity control. The percentage of the ratio between the difference of specific release and spontaneous release to the difference of maximum and spontaneous release was used to calculate specific lysis.

Isograft models of tumor biology. For the MC26 tumor model, $100 \mu \mathrm{l}$ of $5 \times 10^{6}$ cells $/ \mathrm{ml} \mathrm{MC} 26$ cell suspension in PBS was injected subcutaneously into the right flank of BALB $/ \mathrm{c}-E P 2^{-/-}$and wild-type control mice. The size of the tumor was determined by direct measurement of tumor dimensions at 2- to 3-day intervals, using calipers. To quantitate tumor volume the equation, volume $=\left[\right.$ length $\left.\times(\text { width })^{2}\right] \times 0.5$, was used. For the $3 \mathrm{LL}$ tumor model, cell suspension containing $5 \times 10^{5}$ cells in $100 \mu \mathrm{l}$ was injected into tail veins of the wild-type $\mathrm{C} 57 \mathrm{BL} / 6$ or $\mathrm{EP}^{-/-}$mice. The time of death, or when mice became moribund, was recorded and plotted as percentage of survival. The lungs were taken from each animal immediately after death and weighed.

Immunobistochemistry and mouse VEGF immunoassay. Tumor samples from MC26 mice model were fixed with $4 \%$ paraformaldehyde, embedded in paraffin, and sections stained using an anti-CD31 Ab from Research Diagnostics Inc. used at a 1:400 dilution. For the VEGF immunoassay, we followed the protocol described by Marti et al. (26), quantitated using a commercially available immunoassay kit (Quantikine M; R\&D Systems Inc.).

Statistical analysis. Tests of hypotheses concerning the significant difference of the tumor growth between $E P 2^{-/-}$and wildtype controls across different time points were made using the restricted/residual maximum likelihood-based mixed effect model to adjust the intracorrelation effect for the repeated measurements of the tumor volume (in millimeters cubed) from the same mouse. For lifetime data analyses, the two groups (EP2-/- vs. wild-type controls) were compared for survival with
Kaplan-Meier estimates and log-rank tests. For the single time point data, tests of hypotheses between groups were made using either the ANOVA with adjusted leastsquares means or the $\chi$-square test for the interesting continuous or categorical variables, respectively. All tests of significance were two sided, and differences were considered statistically significant when $P$ values were less than 0.05 . All data were expressed as means \pm SE. SAS version 8.0 was used for all analyses (SAS Institute Inc., Carey, North Carolina, USA).

\section{Results}

Attenuated tumor growth and prolonged survival in EP2-/mice. To determine whether the EP2 receptor is important in the escape of tumors from host immune responses, we examined whether tumor growth was suppressed in $\mathrm{EP}^{-/-}$mice relative to wild-type control animals. The syngeneic colorectal cancer cell line MC26 was chosen because it exhibits constitutive COX-2 expression. MC26 cells were injected subcutaneously into wild-type or EP2-null BALB/c mice. Colorectal cancer cells grew rapidly as solid tumors in the wildtype host, whereas the tumor growth, including tumor volume and growth rate, were significantly attenuated in $E P 2^{--}$hosts $(P<0.05)$ (Figure 1a). Similar results were observed with C57BL/6-derived Lewis lung carcinoma (3LL) in $E 2^{--} \mathrm{C} 57 \mathrm{BL} / 6$ animals. The survival of $E P 2^{-/-}$mice was significantly prolonged compared with wild-type control animals after i.v. injection of $3 \mathrm{LL}$ cells (Figure 1b). Terminal lung weight from wild-type mice was also significantly higher than that of $E P 2^{-/-}(875$ $\mathrm{mg}$, wild type; $557 \mathrm{mg}, E P 2^{-/-}, P<0.05$ ).

Tumor angiogenesis was not different between tumors from $E P 2^{-1-}$ and wild-type animals. It has been observed that tumor vascularity was decreased in tumors transplanted into COX-2-deficient mice (2), and EP2-mediated signals have been implicated in tumor angiogenesis

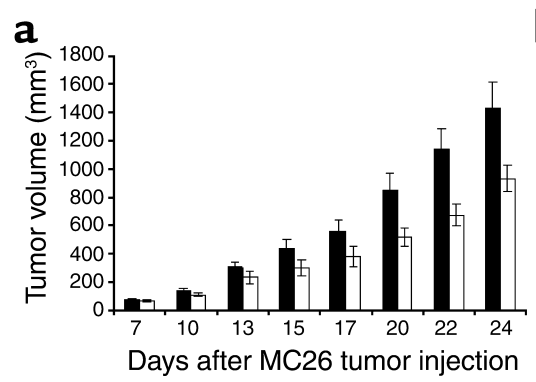

b

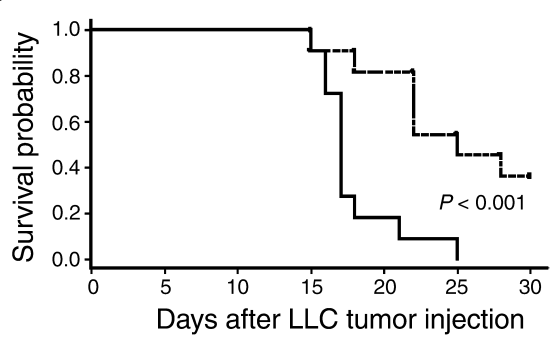

Figure 1

Decreased tumor size and increased host survival in EP2-/- mice. (a) Tumor growth of MC26 was significantly attenuated in $E P 2^{-/-}$host mice. A cell suspension containing $5 \times 10^{5} \mathrm{MC26}$ cells in $100 \mu \mathrm{l}$ PBS was injected subcutaneously into the right flank of EP2-/- (white bars) and wild-type mice (black bars) in the BALB/c background. Tumor volume was determined by direct measurement of tumor dimensions, using calipers. Results are presented as the mean tumor volume $\pm \operatorname{SEM}$ ( $n=34$ for both EP2 $2^{-/-}$and wild-type mice). (b) Survival plot of EP2-/- and wildtype mice after $3 \mathrm{LL}$ tail vein injection. Cell suspensions of $3 \mathrm{LL}$ containing $5 \times 10^{5}$ cells in $100 \mu \mathrm{l}$ PBS were injected into the tail vein of $E P 2^{-/-}$(dashed line) and wild-type mice (solid line) on the C57BL/ 6 background ( $n=11$ for both EP2-/- and wild-type mice). The time of death, or when mice became moribund, was recorded and plotted as percentage of survival. 
a
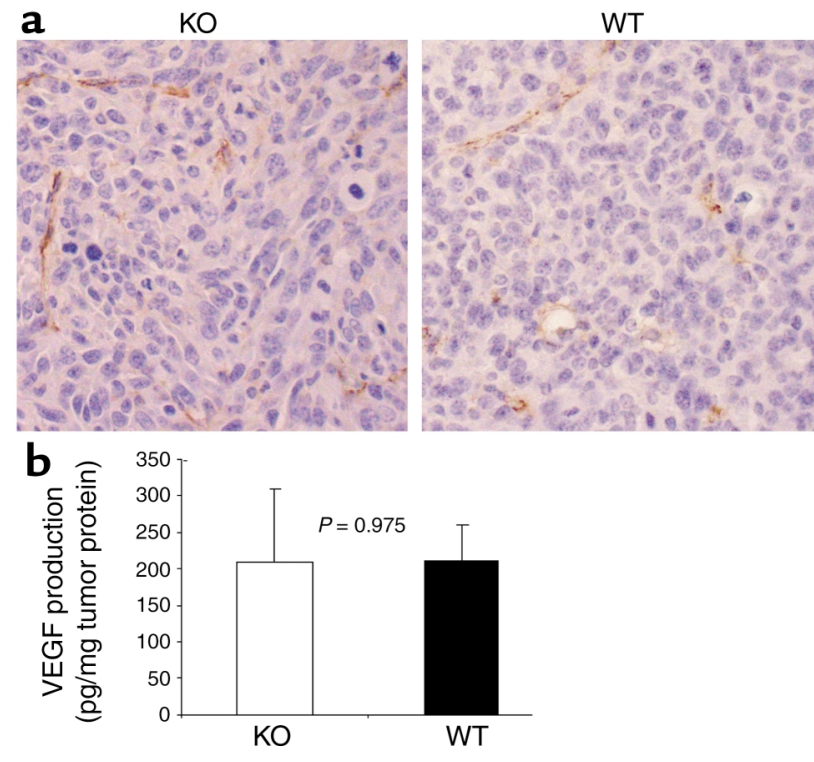

Figure 2

Analysis of tumor angiogenesis in $E P 2^{-/-}$and wild-type animals. (a) Staining of MC26 tumor sections with anti-CD31 Ab. Tumor samples were fixed with $4 \%$ paraformaldehyde, embedded in paraffin, and cut and stained using an anti-CD31 Ab. Blood vessels were visualized by the brown color of the CD31-labeled endothelial cells $(\times 400)$. Slides were lightly counterstained with Mayer's hematoxylin. (b) VEGF concentration of tumors from $E P 2^{-/-}$and wild-type mice. Tumor tissue lysates were prepared by homogenization. Mouse VEGF was quantitated using ELISA. Data show VEGF production in pg per milligram of tumor proteins. Three mice each of $E P 2^{-/-}$and wild type were used, with triplicates for each sample in the immunoassay.

(19). To determine whether the reduced tumor growth rate in the $\mathrm{EP}^{-/-}$background could be explained by effects in tumor angiogenesis, we stained tumor samples from $\mathrm{EP2}^{-/-}$and wild-type mice with anti-CD31 Ab, a marker for endothelial cells. Blood vessels were counted in more than ten high-power fields from four independent MC26 tumors from each background in a blinded evaluation by two independent pathologists. No difference in blood vessel number was observed, with an average of $6.56 \pm 0.76$ and $7.31 \pm 1.5$ vessels per $\times 400$ field $(P=0.498)$ observed in tumors from $E P 2^{-/-}$and wild-type mice, respectively (Figure 2a). Similarly, no difference in CD31 staining was observed in solid tumors in the $3 \mathrm{LL}$ model (data not shown). In addition, we observed no difference in VEGF levels in tumor lysates from $E P 2^{-/-}$and wild-type mice (Figure $2 \mathrm{~b}, P=0.975$ ). These data suggest that alterations in angiogenesis or angiogenic drive do not explain the observed differences in tumor growth between wild-type and $E P 2^{-/-}$mice.

T cell-intrinsic function in EP2-null mice. We hypothesized that the differences in tumor growth rates could be explained by EP2 receptor-mediated immune suppression of the host antitumor response. $\mathrm{PGE}_{2}$ might directly affect $\mathrm{T}$ cell function through the EP2 receptor. To test whether disruption of the $\mathrm{EP} 2$ receptor caused a change in $\mathrm{T}$ cell-elaborated cytokine production, $\mathrm{T}$ cells were isolated from wildtype or EP2-null mice, differentiated into Th1 or Th2 type, and cytokine production was measured after restimulation. The Th1/Th2 cytokine ratio was indistinguishable for both genetic backgrounds (Table 1). We next tested whether the ability of EP2-null T cells to respond to a specific peptide antigen was altered. $\mathrm{BALB} / \mathrm{c}$ EP2-null mice were crossed with DO11.10 mice, which bear an ovalbumin-specific TCR transgene (22). T cells isolated from DO11.10 mice that were $E P 2^{+/+}, E P 2^{+/-}$, or $E P 2^{-/-}$receptor null were stimulated with the wild-type DCs pulsed with the ovalbumin peptide antigen. Ovalbumin peptide-pulsed (OVA 323-339) DCs stimulated $\mathrm{EP}^{+/+}, \mathrm{EP}^{+/-}$, and $E P 2^{-/-} \mathrm{T}$ cells in a dose-dependent manner. There was no enhancement of the proliferation of EP2-null T cells, and, in fact, loss of the EP2 receptor decreased the ability of ovalbumin-specific $T$ cells to respond to antigen (Figure 3). Taken together, these data indicate that the absence of the EP2 receptor does not appear to enhance intrinsic $\mathrm{T}$ cell function, and, if anything, EP2-null T cell function may be suppressed under some conditions. When bone marrow cells from wild-type or EP2-null BALB/c mice were transplanted into MHC class I congenic BALB.B mice, the resulting CD8 CTL from either background did not have substantially different cytolytic activity against the recipient MHC (data not shown), further indicating that the observed effects are unlikely to be due to an alteration in $\mathrm{T}$ cell-intrinsic function.

$P G E_{2}$ suppresses DC differentiation by the EP2 receptor. We next assessed the role of the EP2 receptor in DCs. EP2 receptor mRNA is expressed in wild-type bone marrow progenitor cells but not in progenitors derived from $E P 2^{-/-}$mice (Figure 4a). $\mathrm{PGE}_{2}$ has been shown to increase IL-10 production and decrease class II expression on DCs, although the PG receptor mediating these effects was not identified $(16,17)$. We therefore sought to determine whether $\mathrm{PGE}_{2}$ might act directly on DC differentiation and function through EP2-evoked responses in this system. DCs were cultured from bone marrow progenitors in medium containing varying concentrations of $\mathrm{PGE}_{2}$. On day 9 or 10, DCs were analyzed by FACS for the presence of CD11c, a well-defined DC differentiation marker. While we observed a PGE 2 -mediated dosedependent decrease in the number of cells expressing CD11c, no inhibition was observed in $E P 2^{-/-}$animals at concentrations up to $1,000 \mathrm{nM}$ (Figure 4b). We observed

\section{Table 1}

Cytokine levels in Th1 and Th2 cells derived from wild-type or EP2null mice

\begin{tabular}{lcccc}
\hline & Wild type & Wild type & EP2-/- & $E P 2^{-/-}$ \\
& Th1 cells & Th2 cells & Th1 cells & Th2 cells \\
IL-4 pg/ml & ND & 16,565 & ND & 14,723 \\
IFN- $\gamma$ pg/ml & 195,600 & 919 & 183,400 & 2,317 \\
IFN- $\gamma / \mathrm{lL}-4$ ratio & \multicolumn{2}{c}{11.8} & \multicolumn{2}{c}{12.5} \\
\hline
\end{tabular}




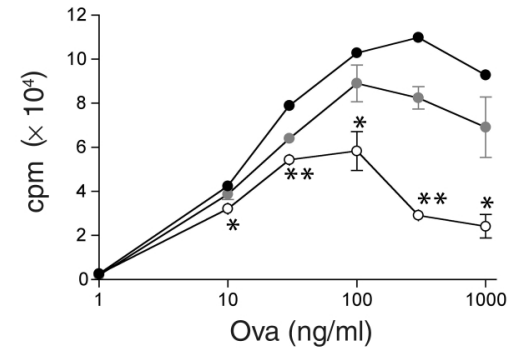

Figure 3

OVA MLR T cell proliferation in wild-type and EP2-null cells. DCs were pulsed with the indicated quantity of OVA 323-339 and used to stimulate T cells bearing the OVA-specific TCR (DO11.10). Proliferation of T cells was measured by ${ }^{3}[\mathrm{H}]$ thymidine uptake. Wildtype $T$ cells are represented by filled symbols, EP2 heterozygotes by shaded symbols, and EP2-null T cells by open symbols. The data are the mean \pm SEM of triplicate measurements. ${ }^{*} E P 2$-null value was significantly different from wild-type value $(P<0.01)$. ${ }^{*}$ EP2-null value is significantly different from wild-type value $(P<0.001)$.

similar effects using H-2 IA ${ }^{\mathrm{d}}$, a MHC class II molecule; CD86, which reflects DC function and influences antigen-presenting and tumor-antigen cross priming; and CD40, which transmits activating signals to immature DCs. In the wild-type control mice, the number of cells expressing each of these markers was significantly decreased $(P<0.05)$ at day 9 in the presence of $100 \mathrm{nM}$ $\mathrm{PGE}_{2}$, while there was no effect on DCs derived from $E P 2^{-/-}$mice (Figure 4). The concentration of $\mathrm{PGE}_{2}$ we used in these studies is similar to the in vitro threshold concentration reported in other studies (27) and to concentrations observed in tumors in Table 2. Incubation of DCs with the EP2 subtype selective agonist butaprost yielded essentially similar results (data not shown).

\section{Figure 4}

Inhibition of expression of DC markers by $\mathrm{PGE}_{2}$. (a) Characterization of EP2 receptor expression in bone marrow progenitors of wild-type and $E P 2^{-1-}$ mice. Total RNA was extracted from bone marrow progenitors, and cDNA was amplified by RT-PCR. Primers specific for the EP2 receptor were used. Lane 1, DNA marker; lane 2, wild type; lane $3, E P 2^{-/-}$; lane 4 and 5 , no reverse transcriptase control for wild type

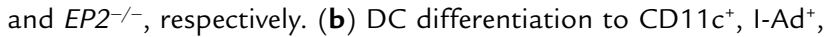
$\mathrm{CD}^{2} 6^{+}$, and $\mathrm{CD} 40^{+}$cells was significantly inhibited after $\mathrm{PGE}_{2}$ treatment. Bone marrow progenitors from $E P 2^{-/-}$(white bars) and wildtype (black bars) mice were cultured in GM-CSF/IL-4 medium with increasing concentrations of $\mathrm{PGE}_{2}$. At day 9, FACScan flow cytometer was used to analyze the phenotype of DCs. The percentage of expression is normalized relative to untreated cells from wild-type mice. Each surface marker was plotted on a bar graph as shown in $\mathbf{b}$. Graphs depict: (c) CD11c; (d) CD86; (e) MHC class II; and (f) CD40. Untreated wild-type cells (black bars), wild-type treated with $100 \mathrm{nM}$ $\mathrm{PGE}_{2}$ (gray bars), untreated $E P 2^{-/-}$cells (white bars), EP2 $2^{-/-}$cells treated with $100 \mathrm{nM} \mathrm{PGE}_{2}$ (dark gray bars). The $\mathrm{PGE}_{2}$ dose response for $\mathrm{CD} 11 \mathrm{c}$ expression is a representative experiment from two independent experiments with similar results. All others are from three to four independent experiments. For each graph there is a statistically significant difference between wild-type/no $\mathrm{PGE}_{2}$ and wild-type/PGE $(P<0.05)$; there are no statistically significant differences between $E P 2^{-/-} /$no $\mathrm{PGE}_{2}$ and $E P 2^{-/-} / \mathrm{PGE}_{2}(P>0.05)$.
$P G E_{2}$ inhibits DC function through the EP2 receptor. We further investigated the ability of $\mathrm{PGE}_{2}$-treated DCs to stimulate $T$ cell proliferation using allogeneic MLR assays. $\mathrm{BALB} / \mathrm{c}$-derived DCs (day 9) were cultured in presence of varying concentrations of $\mathrm{PGE}_{2}$ and mixed with $\mathrm{C} 57 \mathrm{BL} / 6$ wild-type $\mathrm{T}$ cells in the absence of $\mathrm{PGE}_{2} . E P 2^{-/-} \mathrm{DCs}$ exhibited increased $\mathrm{T}$ cell stimulation after culture with 1-50 nM PGE 2 , while DCs from wild-type mice showed decreased stimulation (Figure 5). At concentrations of $100 \mathrm{nM} \mathrm{PGE}_{2}$, T cell proliferation stimulated by wildtype DCs was significantly decreased $(P=0.03)$, whereas $\mathrm{T}$ cell proliferation induced by DCs from $\mathrm{EP}^{-/-}$mice was unaffected. Thus, EP2 receptor is critical for $\mathrm{PGE}_{2-}$ induced suppression of DC-mediated T cell activation. At doses of $1 \mu \mathrm{M} \mathrm{PGE} 2$, suppression MLR was observed in both wild-type $(P<0.003)$ and EP2-null mice $(P<0.01)$; however, these effects were observed only at concentrations greater than 100 -fold over the $K_{d}$ for the EP2 receptor where there is significant cross-talk with other eicosanoid receptors (8).

The EP2 receptor plays an important role in DC function in vivo. An essential function of DC is to take up antigen at the tumor site and transport it to regional lymph nodes, where it is presented to T cells. We assessed the number of DCs and T cells in tumor-draining lymph nodes from $E P 2^{-/-}$and wild-type animals. We used the immunogenic BALB/c-derived MC26 $\left(\mathrm{H}_{2} \mathrm{~K}^{\mathrm{d}}\right)$ tumor

a

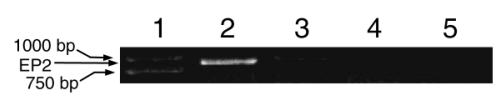

b

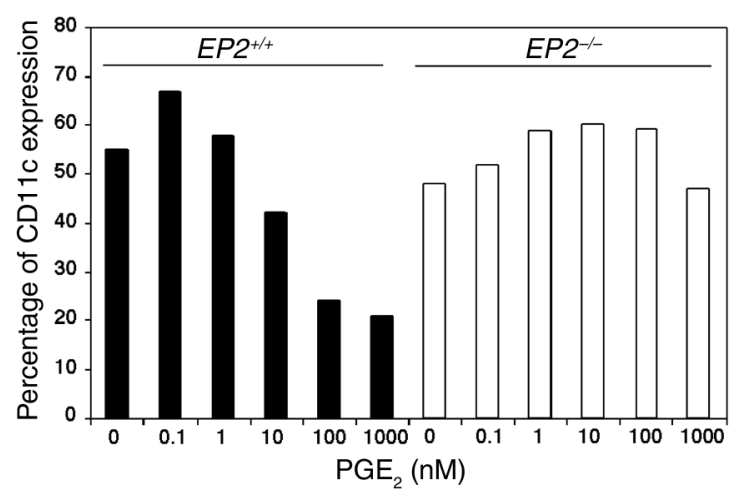

c

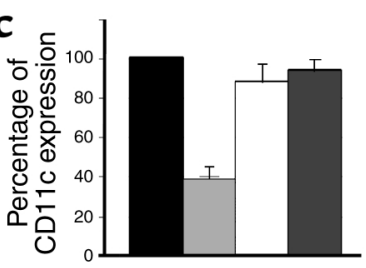

d

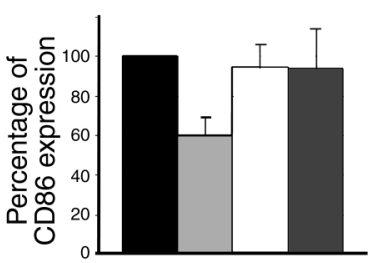

e

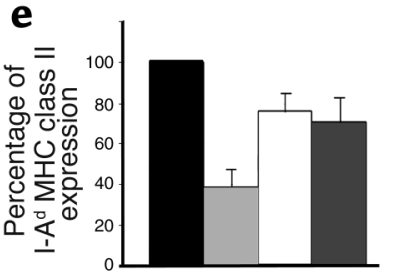

f

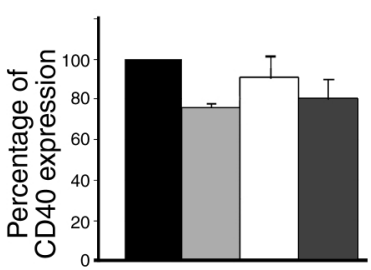


Table 2

$\mathrm{PGE}_{2}$ levels in serum, draining lymph nodes, and tumor tissues

\begin{tabular}{|c|c|c|c|c|}
\hline Sample ${ }^{A}$ & $\begin{array}{c}E P 2^{-/-} \\
\text {tumor bearing }\end{array}$ & $\begin{array}{l}\text { Wild type } \\
\text { tumor bearing }\end{array}$ & $E P 2^{-/-}$ & Wild type \\
\hline Serum (ng/ml) & $4.04 \pm 0.73$ & $3.93 \pm 0.33$ & $2.73 \pm 0.49$ & $3.54 \pm 0.54$ \\
\hline Draining lymph node (ng/g) & $463 \pm 107$ & $731 \pm 238$ & $612 \pm 319$ & $487 \pm 65$ \\
\hline Tumor tissue (ng/g) & $435 \pm 59$ & $558 \pm 140$ & & \\
\hline
\end{tabular}

${ }^{A}$ Average $\mathrm{PGE}_{2}$ concentration $\pm \mathrm{SEM}$ from three to four mice.

model, which has been proposed to express a novel, as yet unidentified, tumor-associated antigen $(28,29)$. These cells express MHC class I (30) and COX-2 (R. Dubois, personal communication). Consistent with improved survival and DC function in the knockout animals, we found that there were significantly more $\mathrm{DCs}, \mathrm{CD}^{+}$, and $\mathrm{CD}^{+} \mathrm{T}$ cells from draining lymph nodes of tumor-bearing $\mathrm{EP}^{-/-}$mice than tumor-bearing wild-type mice, consistent with improved DC function in $\mathrm{EP}^{-/-}$animals (Figure 6). This increase in cell number, however, was observed only in tumor-bearing animals, with no differences in DC or T cell numbers observed between genotypes in normal lymph nodes. Importantly, the number of DCs was significantly suppressed in the draining lymph nodes of wild-type tumor-bearing animals as compared with non-tumorbearing animals, in contrast to the higher DC numbers observed in the EP2-null tumor-bearing animals as compared with EP2-null controls. These data are consistent with a tumor immunosuppressive effect in wildtype but not EP2-null animals. Supporting the notion that the immune response differences were tumor induced, no differences were observed in the fraction of activated $\mathrm{CD}^{+}, \mathrm{CD}^{+}, \mathrm{NK} T$ cells or NK cells in normal lymph nodes using CD69 as a marker of early activation (data not shown).

There was no significant difference in $\mathrm{PGE}_{2}$ concentration from tumors, draining lymph nodes, or serum between tumor-bearing $E P^{-{ }^{--}}$and wild-type mice $(P>0.05$; see Table 2$)$. The improvement noted in DC function in $\mathrm{EP}^{-/-}$mice is thus unlikely to be mediated through indirect regulation of $\mathrm{PGE}_{2}$ production and its action on other receptors.

To establish the functional significance of increased $\mathrm{DC}$ and $\mathrm{CD}^{+} \mathrm{T}$ cell numbers, the specificity of tumorspecific CTLs in a syngeneic tumor model was determined. $E P 2^{-/-}$and wild-type mice were immunized with irradiated MC26 cells. Spleen cells from MC26immunized $\mathrm{EP}^{-/-}$mice exhibited specific cytotoxicity for MC26 tumor cells, whereas spleen cells from MC26-immunized wild-type mice showed no detectable CTL activity (Figure 7). The R8 cell line, which expresses both $\mathrm{H} 2^{\mathrm{b}}$ and $\mathrm{H} 2^{\mathrm{d}}$ MHC class I molecules, used as a control for target cell specificity, was not lysed by MC26-specific CTLs (data not shown). Furthermore, RMA-S cells, which do not express MHC class I molecules and which form targets for NK cells, were also not lysed by MC26-specific CTLs (data not shown), demonstrating that the effector function of
MC26-immunized spleen cells is not due to NK cell activity. Thus the MC26-specific immune reaction observed is CTL mediated, which is MHC restricted and tumor antigen specific.

\section{Discussion}

The overproduction of COX-2 and its major metabolite $\mathrm{PGE}_{2}$ have been found in many human cancers, including colorectal tumors, lung cancer (31-34), and cancer cell lines $(5,35,36)$. Tumor-infiltrating macrophages also produce high levels of $\mathrm{PGE}_{2}$ (37). Our data demonstrate that the PG receptor EP2 plays a key role in $\mathrm{PGE}_{2}$-induced inhibition of DC differentiation and function and cancer-associated immunodeficiency. Two major host-response mechanisms known to be affected by $\mathrm{PGE}_{2}$ are tumor angiogenesis and immune responses. We found no differences in either microvessel counts or tumor tissue VEGF levels between tumor samples of $\mathrm{EP}^{-/-}$and wild-type mice, rendering it unlikely that significant differences in angiogenesis underlie the observed differences in tumor growth. Multiple assays for $\mathrm{T}$ cell function demonstrated no significant differences between wildtype and EP2-null mice. In contrast, we observed that

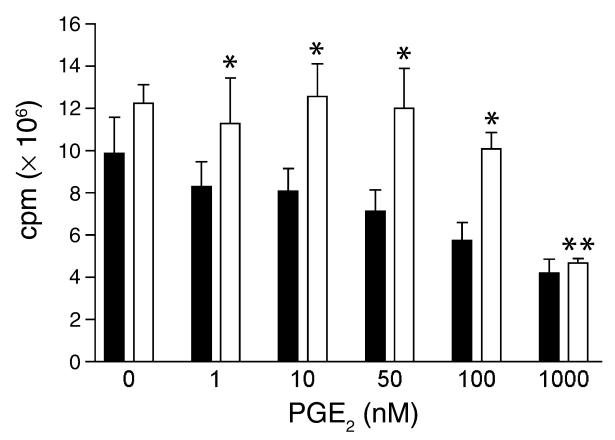

Figure 5

$E P 2^{-/-} \mathrm{DCs}$ are refractory to mediated $\mathrm{PGE}_{2}$ functional inhibition in vitro. $P G E_{2}$ induced inhibition of $D C$ function signals through the EP2 receptor, shown by allogenic MLR. Day 9 DCs from $E P 2^{-/-}$ (white bars) and wild-type (black bars) mice on the BALB/c background, treated with $\mathrm{PGE}_{2}$, were irradiated with $20 \mathrm{~Gy}$, plated in triplicate, and incubated with $\mathrm{T}$ cells from wild-type mice with C57BL/ 6 background for $72 \mathrm{~h}$. All cultures were pulsed with $1 \mu \mathrm{M}$ $\left[{ }^{3} \mathrm{H}\right]$ thymidine $18 \mathrm{~h}$ before harvesting. Incorporation of $\left[{ }^{3} \mathrm{H}\right]$ thymidine was counted using a liquid scintillation counter and expressed as counts per minute. ${ }^{*} E P 2$-null value was significantly different from wild-type value $(P<0.02) .{ }^{*} E P 2$-null value is significantly different from EP2-null untreated control $(P<0.01)$. 
a

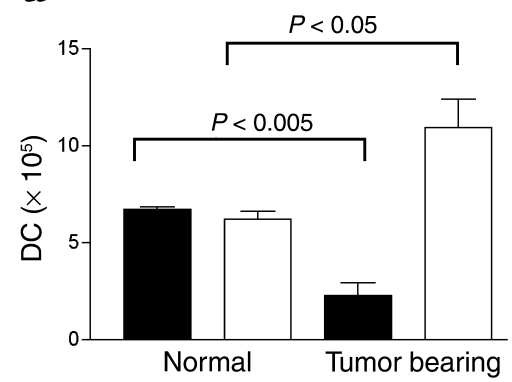

b

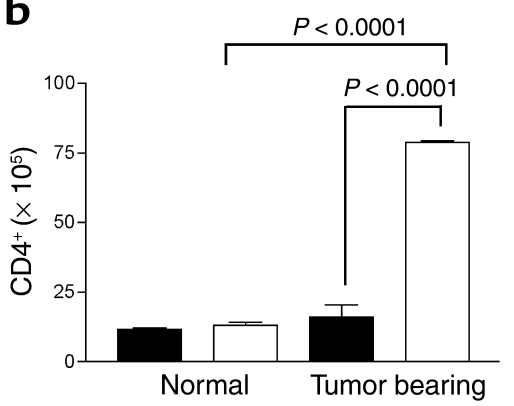

c

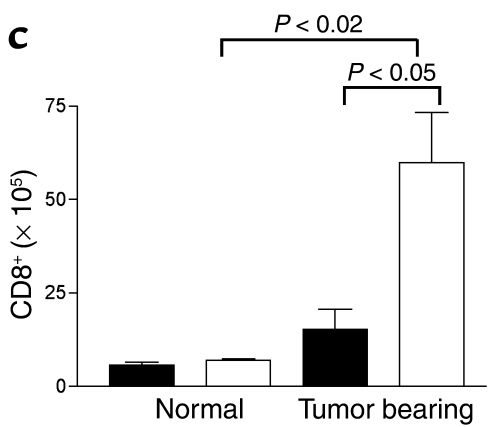

Figure 6

Increased DC numbers draining lymph nodes of EP2-null mice. The number of (a) DCs, (b) CD4 ${ }^{+}$, and (c) CD8 ${ }^{+}$T cells obtained from draining lymph nodes of normal or tumor-bearing $E P 2^{-/-}$mice (white bars) is increased compared with wild-type control mice (black bars). For tumor-bearing mice, single-cell suspensions from inguinal lymph nodes from tumor-bearing $E P 2^{-/-}$and wild-type mice were prepared 21 days after MC26 inoculation, at which time we observed the maximum tumor-growth difference. Cells were labeled with PE-conjugated anti-I-A CD11c Ab's, anti-CD4, and anti-CD8 Ab's. Cells were then washed and analyzed using a FACScan flow cytometer. The total number of positive cells per mouse is shown. Each data point is from three mice.

$\mathrm{PGE}_{2}$ dramatically inhibits DC differentiation and function of bone marrow-derived DC cultures in vitro and that this effect was nearly eliminated in cells from $E P 2^{-/-}$animals. Furthermore, the number of DCs, $\mathrm{CD}^{+}$, and $\mathrm{CD}^{+} \mathrm{T}$ cells from draining lymph nodes of tumor-bearing $\mathrm{EP}^{---}$mice were significantly increased compared with tumor-bearing wild-type control mice. Indeed, DC numbers were suppressed in draining lymph nodes of wild-type tumor-bearing mice, consistent with a tumor immunosuppressive effect (Figure 6). Perhaps most significantly, tumor-specific CTLs were observed only in immunized $\mathrm{EP}^{-/-}$animals and not in controls. Together, these data indicate that $\mathrm{PGE}_{2}$ acts through the EP2 receptor to inhibit the host immune response to tumors.

The COX-derived metabolites have pleiotropic effects on tumor growth. Reduced tumor growth has been noted in COX-2/- animals $(2,38)$, suggesting a tumorpromoting effect of COX-2 products. However, mice with pulmonary overexpression of prostacyclin synthase exhibited significantly reduced lung tumor incidence (39). Manipulation of PG metabolism downstream from COX produces more profound cancer reduction than COX inhibition alone and could be the basis for new approaches to understanding the pathogenesis and prevention of cancer. Our observation that tumor growth is reduced by $50 \%$ in $\mathrm{EP}^{-/-}$mice is particularly significant in light of the fact that the EP receptor family contains four receptors, designated EP1, EP2, EP3, and EP4, encoded by distinct genes. While the EP2 receptor plays a major role in the host response to tumors, other receptors, e.g., EP4, may also play a role through mechanisms that are distinct from the one described here.

In addition to the specific link between EP2 and antitumor immunity revealed by our study, COX products can play a role in pathological angiogenesis. It has been reported that vascular density is decreased in tumors from a wild-type background transplanted into $\mathrm{COX}-2^{-/-}$mice (2), and EP2-mediated signals are involved in tumor angiogenesis in tumors developing in the $A p c^{\Delta 716}$ mouse model (19). Mechanisms underlying the differences observed in the $A p c^{\Delta 716}$ model and the transplantable tumors used in the current studies are not clear, but may be the result of specific profiles of proangiogenic factors or tumor antigenicity in a particular tumor model.

In vitro evidence for a role for the EP2 receptor in antigen presentation has been reported by Nataraj et al. (13), who found a decrease in the ability of $\mathrm{PGE}_{2}$ to inhibit $E P 2^{-/-}$macrophage in stimulating an allogeneic MLR. We have confirmed and extended this MLR finding with purified DCs. Moreover, we observed that the differentiation of DCs grown from wild-type bone marrow progenitors is significantly inhibited when cultured in vitro in the presence of $\mathrm{PGE}_{2}$, in contrast to progenitors from $\mathrm{EP}^{-/-}$mice, demonstrating effects of $\mathrm{PGE}_{2}$ on progenitors of that lineage. Previous studies have shown that elevated $\mathrm{PGE}_{2}$ promotes type 2 cytokine production in human $\mathrm{T}$ helper cells by impair-

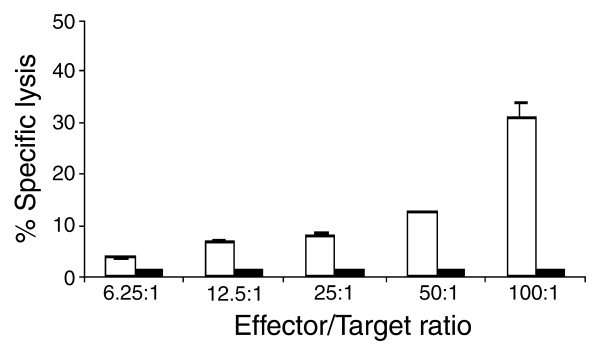

Figure 7

CTL function is elevated in EP2-/- mice. Spleen cells from MC26immunized $E P 2^{-/-}$(white bars) and wild-type mice (black bars) were cocultured with irradiated MC26 cells for 4 days. The cells were then washed and incubated with different ratios of ${ }^{51} \mathrm{Cr}$-labeled MC26 cells. After a 6-h incubation, supernatants were harvested and radioactivity was measured using a gamma counter. The maximum and spontaneous release and the percentage of specific ${ }^{51} \mathrm{Cr}$ release were determined. Data shown are representative of two independent experiments with similar results. 
ing IL-12 response of DCs $(40,41)$. Other factors secreted by tumors dramatically affect DC maturation from precursors, including VEGF (42). The mechanism of the $\mathrm{PGE}_{2}$ effect on DCs may thus involve alterations in local or systemic cytokine production.

Most importantly, consistent with a role for the EP2 receptor in DC trafficking and differentiation, as well as function, we observed increased numbers of DCs and $\mathrm{CD}^{+}$and $\mathrm{CD}^{+} \mathrm{T}$ cells in tumor-draining lymphoid organs. Moreover, specific antitumor immunity in $E P 2^{-/-}$mice was increased dramatically compared with controls. It is well known that DC function is critical for $\mathrm{T}$ lymphocyte-mediated immune responses to tumors, both in animal models and humans. Previous studies suggested that defective antigen presentation by DCs appears to be a major determinant for CTLs' nonresponsiveness to peptide antigens in tumor-bearing mice. Addition of control peptide-pulsed DCs restored specific tumor lysis (43). Inadequate presentation of tumor antigens by host professional APCs, including DCs, is thus a major mechanism for the escape of tumors from the host immune system (42). Cross priming of tumor antigens by host APCs, especially DCs, the most potent APCs, through the MHC class I and class II pathway has been postulated to be a necessary and efficient mechanism to induce protective CTL responses (44-49). Furthermore, specific inhibition of COX-2 or $\mathrm{PGE}_{2}$ activity with specific $\mathrm{mAb}$ restores antitumor reactivity by altering the balance of IL-10 and IL-12 synthesis (6). PGE 2 is also reported to downregulate IFN- $\gamma$ production and might, in principle, thereby interfere with cell-mediated immunity $(50$, 51). Here we demonstrate that absence of the $\mathrm{PGE}_{2} \mathrm{EP} 2$ receptor prevents the inhibition of DC function and allows induction of specific immunity against the tumor. Thus $\mathrm{PGE}_{2}$ secreted by tumor cells is likely responsible for this escape by signaling through the EP2 receptor on immune cells.

In conclusion, the EP2 receptor plays an important role in inhibiting the host reaction to tumor growth through diminished immune responses caused by defective DC differentiation and function. These data suggest that the development of selective antagonists against the EP2 receptor has the potential to improve host antitumor immunity. EP2-receptor antagonists may be more specific than the use of COX-2 inhibitors and avoid inhibition of products such as prostacyclin that may be beneficial in the antitumor response.

\section{Acknowledgments}

We thank the Vanderbilt University School of Medicine Mouse Pathology and Immunohistochemistry Core Labs for providing immunohistochemical staining of CD31. We appreciate Roy Jensen and Adriana Gonzales for the evaluation of CD31 staining. This work was supported by grants from the Vanderbilt-Ingram Cancer Center, as well as the NIH: GM-15431 (R.M. Breyer and J.D. Morrow), DK-46205 (R.M. Breyer), CA-77839 and DK-48831 (J.D. Morrow), and 1P50 CA 90949-01
(D.P. Carbone). Jason D. Morrow is the recipient of a Burroughs Wellcome Fund Clinical Scientist Award in Translational Research. We thank John Oates and Larry Marnett for critical reading of the manuscript.

1. Liu, C.H., et al. 2001. Overexpression of cyclooxygenase- 2 is sufficient to induce tumorigenesis in transgenic mice. J. Biol. Chem. 276:18563-18569.

2. Williams, C.S., Tsujii, M., Reese, J., Dey, S.K., and DuBois, R.N. 2000. Host cyclooxygenase-2 modulates carcinoma growth. J. Clin. Invest. 105:1589-1594.

3. Tsujii, M., et al. 1998. Cyclooxygenase regulates angiogenesis induced by colon cancer cells. Cell. 93:705-716.

4. Snijdewint, F.G., Kalinski, P., Wierenga, E.A., Bos, J.D., and Kapsenberg, M.L. 1993. Prostaglandin E2 differentially modulates cytokine secretion profiles of human T helper lymphocytes. J. Immunol. 150:5321-5329.

5. Huang, M., et al. 1998. Non-small cell lung cancer cyclooxygenase-2dependent regulation of cytokine balance in lymphocytes and macrophages: up-regulation of interleukin 10 and down-regulation of interleukin 12 production. Cancer Res. 58:1208-1216.

6. Stolina, M., et al. 2000. Specific inhibition of cyclooxygenase 2 restores antitumor reactivity by altering the balance of IL-10 and IL-12 synthesis. J. Immunol. 164:361-370.

7. Narumiya, S., Sugimoto, Y., and Ushikubi, F. 1999. Prostanoid receptors: structures, properties, and functions. Physiol. Rev. 79:1193-1226.

8. Breyer, R.M., Bagdassarian, C.K., Myers, S.A., and Breyer, M.D. 2001. Prostanoid receptors: subtypes and signaling. Annu. Rev. Pharmacol. Toxicol. 41:661-690.

9. Tilley, S.L., Coffman, T.M., and Koller, B.H. 2001. Mixed messages: modulation of inflammation and immune responses by prostaglandins and thromboxanes. J. Clin. Invest. 108:15-23. doi:10.1172/JCI200113416.

10. Fedyk, E.R., and Phipps, R.P. 1996. Prostaglandin E2 receptors of the EP2 and EP4 subtypes regulate activation and differentiation of mouse B lymphocytes to IgE-secreting cells. Proc. Natl. Acad. Sci. U. S. A. 93:10978-10983

11. Rocca, B., et al. 1999. Distinct roles of prostaglandin $H$ synthases 1 and 2 in T-cell development. J. Clin. Invest. 103:1469-1477.

12. Rocca, B., Spain, L.M., Ciabattoni, G., Patrono, C., and FitzGerald, G.A. 1999. Differential expression and regulation of cyclooxygenase isozymes in thymic stromal cells. J. Immunol. 162:4589-4597.

13. Nataraj, C., et al. 2001. Receptors for prostaglandin $E_{2}$ that regulate cellular immune responses in the mouse. J. Clin. Invest. 108:1229-1235. doi:10.1172/JCI200113640.

14. Strong, V.E., et al. 2001. Prostaglandin E2 receptors EP2 and EP4 are down-regulated in human mononuclear cells after injury. Surgery. 130:249-255

15. Li, X., et al. 2000. Knockout of the murine prostaglandin EP2 receptor impairs osteoclastogenesis in vitro. Endocrinology. 141:2054-2061.

16. Harizi, H., Juzan, M., Grosset, C., Rashedi, M., and Gualde, N. 2001. Dendritic cells issued in vitro from bone marrow produce PGE(2) that contributes to the immunomodulation induced by antigen-presenting cells. Cell Immunol. 209:19-28.

17. Harizi, H., Juzan, M., Pitard, V., Moreau, J.F., and Gualde, N. 2002. Cyclooxygenase-2-issued prostaglandin $\mathrm{E}(2)$ enhances the production of endogenous IL-10, which down-regulates dendritic cell functions. J. Immunol. 168:2255-2263.

18. Rozic, J.G., Chakraborty, C., and Lala, P.K. 2001. Cyclooxygenase inhibitors retard murine mammary tumor progression by reducing tumor cell migration, invasiveness and angiogenesis. Int. J. Cancer. 93:497-506.

19. Sonoshita, M., et al. 2001. Acceleration of intestinal polyposis through prostaglandin receptor EP2 in ApcDelta716 knockout mice. Nat. Med. 7:1048-1051.

20. Seno, H., et al. 2002. Cyclooxygenase 2- and prostaglandin $\mathrm{E}(2)$ receptor $\mathrm{EP}(2)$-dependent angiogenesis in $\mathrm{Apc}($ Delta716) mouse intestinal polyps. Cancer Res. 62:506-511.

21. Kennedy, C.R., et al. 1999. Salt-sensitive hypertension and reduced fertility in mice lacking the prostaglandin EP2 receptor. Nat. Med. 5:217-220.

22. Murphy, K.M., Heimberger, A.B., and Loh, D.Y. 1990. Induction by antigen of intrathymic apoptosis of CD4+CD8+TCRlo thymocytes in vivo. Science. 250:1720-1723.

23. Youn, J., et al. 1998. In vivo function of an interleukin 2 receptor beta chain (IL-2Rbeta)/IL-4Ralpha cytokine receptor chimera potentiates allergic airway disease. J. Exp. Med. 188:1803-1816.

24. DuBois, R.N., et al. 1994. Cloning and characterization of a growth factor-inducible cyclooxygenase gene from rat intestinal epithelial cells. Am. J. Physiol. 266:G822-G827.

25. Song, H., et al. 2002. Cytosolic phospholipase A2alpha is crucial for 'ontime' embryo implantation that directs subsequent development. Development. 129:2879-2889. 
26. Marti, H.J., et al. 2000. Hypoxia-induced vascular endothelial growth factor expression precedes neovascularization after cerebral ischemia Am. J. Pathol. 156:965-976.

27. Mukutmoni, M., Hubbard, N.E., and Erickson, K.L. 2001. Prostaglandin E(2) modulation of vascular endothelial growth factor production in murine macrophages. Prostaglandins Leukot. Essent. Fatty Acids. 65:123-131.

28. Rodolfo, M., et al. 1994. Cytotoxic T lymphocytes recognize tumor antigens of a murine colonic carcinoma by using different T-cell receptors. Int. J. Cancer. 57:440-447.

29. Griswold, D., and Corbett, T. 1975. A colon tumor model for anticancer agent evaluation. Cancer. 36:2441-2444.

30. Gagandeep, S., et al. 1996. Prodrug-activated gene therapy: involvement of an immunological component in the "bystander effect". Cancer Gene Ther. 3:83-88.

31. Hida, T., et al. 1998. Increased expression of cyclooxygenase 2 occurs frequently in human lung cancers, specifically in adenocarcinomas. Cancer Res. 58:3761-3764.

32. Eberhart, C.E., et al. 1994. Up-regulation of cyclooxygenase 2 gene expression in human colorectal adenomas and adenocarcinomas. Gastroenterology. 107:1183-1188.

33. Sano, H., et al. 1995. Expression of cyclooxygenase- 1 and -2 in human colorectal cancer. Cancer Res. 55:3785-3789.

34. Kargman, S.L., et al. 1995. Expression of prostaglandin G/H synthase-1 and -2 protein in human colon cancer. Cancer Res. 55:2556-2559.

35. Chen, Y., and Hughes-Fulford, M. 2000. Prostaglandin E2 and the protein kinase A pathway mediate arachidonic acid induction of $\mathrm{c}$-fos in human prostate cancer cells. Br. J. Cancer. 82:2000-2006.

36. Chen, W.S., et al. 2001. Tumor invasiveness and liver metastasis of colon cancer cells correlated with cyclooxygenase-2 (COX-2) expression and inhibited by a COX-2-selective inhibitor, etodolac. Int. J. Cancer. 91:894-899.

37. Berens, M.E., Salmon, S.E., and Davis, T.P. 1984. Quantitative analysis of prostaglandins in cell culture medium by high-resolution gas chromatography with electron-capture detection. J. Chromatogr. 307:251-260.

38. Oshima, M., et al. 1996. Suppression of intestinal polyposis in Apc delta716 knockout mice by inhibition of cyclooxygenase 2 (COX-2). Cell. 87:803-809.

39. Keith, R.L., et al. 2002. Manipulation of pulmonary prostacyclin synthase expression prevents murine lung cancer. Cancer Res. 62:734-740.
40. Kalinski, P., Hilkens, C.M., Snijders, A., Snijdewint, F.G., and Kapsenberg, M.L. 1997. Dendritic cells, obtained from peripheral blood precursors in the presence of PGE2, promote Th2 responses. Adv. Exp. Med. Biol. 417:363-367.

41. Kalinski, P., Hilkens, C.M., Snijders, A., Snijdewint, F.G., and Kapsenberg, M.L. 1997. IL-12-deficient dendritic cells, generated in the presence of prostaglandin E2, promote type 2 cytokine production in maturing human naive T helper cells. J. Immunol. 159:28-35.

42. Gabrilovich, D.I., et al. 1996. Production of vascular endothelial growth factor by human tumors inhibits the functional maturation of dendritic cells. Nat. Med. 2:1096-1103.

43. Gabrilovich, D.I., Ciernik, I.F., and Carbone, D.P. 1996. Dendritic cells in antitumor immune responses. I. Defective antigen presentation in tumor-bearing hosts. Cell Immunol. 170:101-110.

44. Carbone, F.R., Kurts, C., Bennett, S.R., Miller, J.F., and Heath, W.R. 1998. Cross-presentation: a general mechanism for CTL immunity and tolerance. Immunol. Today. 19:368-373.

45. Huang, A.Y. 1994. Role of bone marrow-derived cells in presenting MHC class I-restricted tumor antigens. Science. 264:961-965.

46. Yewdell, J.W., Norbury, C.C., and Bennink, J.R. 1999. Mechanisms of exogenous antigen presentation by MHC class I molecules in vitro and in vivo: implications for generating $\mathrm{CD} 8+\mathrm{T}$ cell responses to infectious agents, tumors, transplants, and vaccines. Adv. Immunol. 73:1-77.

47. Jeannin, P., et al. 2000. OmpA targets dendritic cells, induces their maturation and delivers antigen into the MHC class I presentation pathway. Nat. Immunol. 1:502-509.

48. Ossendorp, F., Mengede, E., Camps, M., Filius, R., and Melief, C.J. 1998. Specific T helper cell requirement for optimal induction of cytotoxic $\mathrm{T}$ lymphocytes against major histocompatibility complex class II negative tumors. J. Exp. Med. 187:693-702.

49. Baxevanis, C.N., et al. 2000. Tumor-specific CD4+ T lymphocytes from cancer patients are required for optimal induction of cytotoxic $\mathrm{T}$ cells against the autologous tumor. J. Immunol. 164:3902-3912.

50. Luo, J.S., Kammerer, R., and von Kleist, S. 2000. Comparison of the effects of immunosuppressive factors from newly established colon carcinoma cell cultures on human lymphocyte proliferation and cytokine secretion. Tumour Biol. 21:11-20.

51. Cheng, X., and Lopez, D.M. 1998. CD4+, but not CD8+, T cells from mammary tumor-bearing mice have a down-regulated production of IFN-gamma: role of phosphatidyl serine. J. Immunol. 160:2735-2741. 